\title{
ALGEBRAIC CYCLES ON SEVERI-BRAUER SCHEMES OF PRIME DEGREE OVER A CURVE
}

\author{
Cristian D. GonzÁlez-Avilés
}

\begin{abstract}
Let $k$ be a perfect field and let $p$ be a prime number different from the characteristic of $k$. Let $C$ be a smooth, projective and geometrically integral $k$-curve and let $X$ be a Severi-Brauer $C$-scheme of relative dimension $p-1$. In this paper we show that $C H^{d}(X)_{\text {tors }}$ contains a subgroup isomorphic to $C H_{0}(X / C)$ for every $d$ in the range $2 \leq d \leq p$. We deduce that, if $k$ is a number field, the full Chow ring $C H^{*}(X)$ is a finitely generated abelian group.
\end{abstract}

\section{Introduction.}

Let $k$ be a perfect field with algebraic closure $\bar{k}$ and let $p$ be a prime number different from the characteristic of $k$. Let $C$ be a smooth, projective and geometrically integral $k$-curve. In this paper we study a certain subgroup of $C H^{d}(X)_{\text {tors }}$ for a Severi-Brauer $C$-scheme $q: X \rightarrow C$ of relative dimension $p-1$ and any integer $d$ such that $2 \leq d \leq p$. Let

$$
C H_{0}(X / C)=\operatorname{Ker}\left[C H_{0}(X) \stackrel{q_{*}}{\longrightarrow} C H_{0}(C)\right]
$$

and let $\pi^{*}: C H^{d}(X) \rightarrow C H^{d}(\bar{X})$ be induced by the extension-of-scalars map $\bar{X} \rightarrow X$, where $\bar{X}=X \times_{\operatorname{Spec} k} \operatorname{Spec} \bar{k}$. Then the following holds.

Main Theorem. For any $d$ as above, there exists a canonical isomorphism

$$
\operatorname{Ker}\left[C H^{d}(X) \stackrel{\pi^{*}}{\longrightarrow} C H^{d}(\bar{X})\right] \simeq C H_{0}(X / C) .
$$

Corollary. Assume that $k$ is a number field. Then the Chow ring $C H^{*}(X)$ is a finitely generated abelian group.

The above corollary confirms a well-known conjecture of S.Bloch in a particular case. Previous work on Bloch's conjecture include [3], where $C H^{2}(X)$ is shown to be finitely generated for a certain class of varieties $X$, and [4], where the same result is obtained for $C H_{0}(X)$ when $X \rightarrow C$ is an arbitrary (i.e., not necessarily smooth over $C)$ Severi-Brauer fibration of squarefree index.

\section{Acknowledgements.}

I thank B.Kahn for some helpful comments. I also thank the referee for correcting some inaccuracies and for providing Remark 4.5.

Received by the editors January 29, 2007.

2000 Mathematics Subject Classification. Primary 14C25; Secondary 14C15 .

Key words and phrases. Algebraic cycles, Chow groups, curves, Severi-Brauer schemes.

The author is partially supported by Fondecyt grant 1061209 and Universidad Andrés Bello grant DI-29-05/R. 


\section{Preliminaries.}

Let $k$ be a perfect field, fix an algebraic closure $\bar{k}$ of $k$ and let $\Gamma=\operatorname{Gal}(\bar{k} / k)$. Now let $C$ be a smooth, projective and geometrically integral $k$-curve and let $X$ be a Severi-Brauer scheme over $C[6, \S 8]$ of dimension $m \geq 2$. There exists a proper and flat $k$-morphism $q: X \rightarrow C$ all of whose fibers are Severi-Brauer varieties of dimension $m-1$ over the appropriate residue field [loc.cit.]. We will write $X_{\eta}$ for the generic fiber $X \times_{C} \operatorname{Spec} k(C)$ of $q$ and $A$ for the central simple $k(C)$-algebra associated to $X_{\eta}$. We define

$$
C H_{0}(X / C)=\operatorname{Ker}\left[C H_{0}(X) \stackrel{q_{*}}{\longrightarrow} C H_{0}(C)\right] .
$$

Now let $C_{0}$ be the set of closed points of $C$. The group of divisorial norms of $X / C$ (cf. [8]) is the group

$$
k(C)_{\mathrm{dn}}^{*}=\left\{f \in k(C)^{*}: \forall y \in C_{0}, \operatorname{ord}_{y}(f) \in\left(q_{y}\right)_{*}\left(C H_{0}\left(X_{y}\right)\right)\right\}
$$

where, for each $y \in C_{0}, q_{y}: X_{y} \rightarrow \operatorname{Spec} k(y)$ is the structural morphism of the fiber $X_{y}$. This group is closely related to $C H_{0}(X / C)$ (see [4, Proposition 3.1]). Indeed, there exists a canonical isomorphism

$$
C H_{0}(X / C) \simeq k(C)_{\mathrm{dn}}^{*} / k^{*} \operatorname{Nrd} A^{*} .
$$

Remark 2.1. Fix an integer $d$ such that $1 \leq d \leq m$ and let

$$
C H^{d}(X)^{\prime}=\operatorname{Ker}\left[C H^{d}(X) \stackrel{\pi^{*}}{\longrightarrow} C H^{d}(\bar{X})^{\Gamma}\right],
$$

where $\pi: \bar{X} \rightarrow X$ is the canonical map. A simple transfer argument shows that $C H^{d}(X)^{\prime}$ is a subgroup of $C H^{d}(X)_{\text {tors }}$. Now, since $\bar{X} \rightarrow \bar{C}$ has a section, $\bar{X}$ is a projective bundle over $\bar{C}$. Thus, by [5, Theorem 3.3(b), p.64], there exist isomorphisms

$$
C H^{d}(\bar{X}) \simeq \begin{cases}\mathbb{Z} \oplus C H_{0}(\bar{C}) & \text { if } 1 \leq d \leq m-1 \\ C H_{0}(\bar{C}) & \text { if } \quad d=m .\end{cases}
$$

Therefore, if $J_{C}(k)$ is finitely generated, where $J_{C}$ is the Jacobian variety of $C$ (e.g., $k$ is a number field or $\left.C=\mathbb{P}_{k}^{1}\right)$, then $C H^{d}(X)$ is finitely generated if and only if $C H^{d}(X)^{\prime}$ is finite.

\section{The general method.}

Let $C$ be as above and let $X$ be any smooth, projective and geometrically integral $k$-variety such that there exists a proper and flat morphism $q: X \rightarrow C$ whose generic fiber $X_{\eta}$ is geometrically integral. We have an exact sequence [9]

$$
H^{d-1}\left(X_{\eta}, \mathcal{K}_{d}\right) \stackrel{\delta}{\longrightarrow} \bigoplus_{y \in C_{0}} C H^{d-1}\left(X_{y}\right) \rightarrow C H^{d}(X) \stackrel{j^{*}}{\rightarrow} C H^{d}\left(X_{\eta}\right) \rightarrow 0,
$$

where $j: X_{\eta} \rightarrow X$ is the natural map and the map which we have labeled $\delta$ will play a role later when $k=\bar{k}$. A similar exact sequence exists over $\bar{k}$, and we have two 
natural exact commutative diagrams:

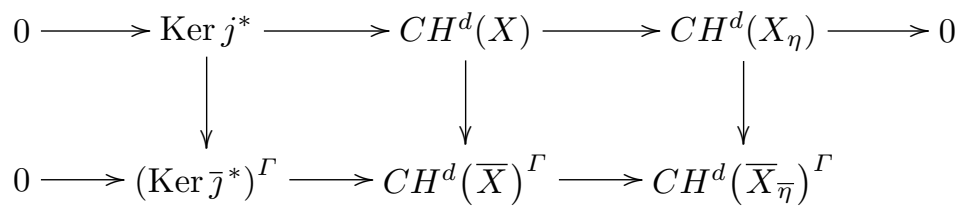

and

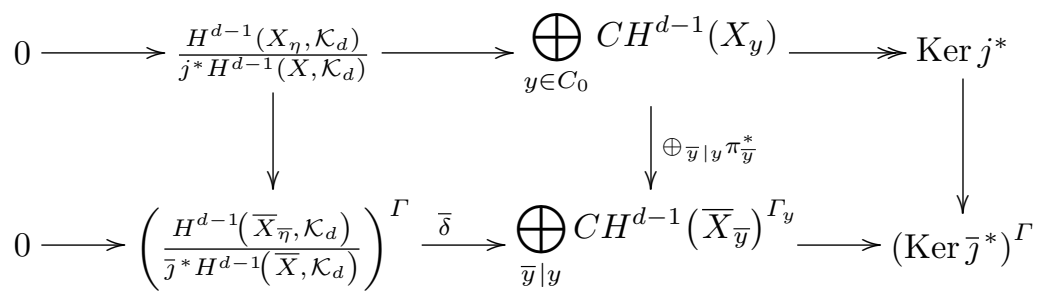

where, for each $y \in C_{0}$, we have fixed a closed point $\bar{y}$ of $\bar{C}$ lying above $y$ and written $\Gamma_{y}=\operatorname{Gal}(\bar{k} / k(y))$. Set

$$
C H^{d}\left(X_{\eta}\right)^{\prime}=\operatorname{Ker}\left[C H^{d}\left(X_{\eta}\right) \rightarrow C H^{d}\left(\bar{X}_{\bar{\eta}}\right)^{\Gamma}\right]
$$

and, for each $y \in C_{0}$,

$$
C H^{d-1}\left(X_{y}\right)^{\prime}=\operatorname{Ker}\left[C H^{d-1}\left(X_{y}\right) \stackrel{\pi_{\bar{y}}^{*}}{\longrightarrow} C H^{d-1}\left(\bar{X}_{\bar{y}}\right)^{\Gamma_{y}}\right] .
$$

Now define

$$
E(\bar{X} / \bar{C})=\text { Coker }\left[\frac{H^{d-1}\left(X_{\eta}, \mathcal{K}_{d}\right)}{j^{*} H^{d-1}\left(X, \mathcal{K}_{d}\right)} \longrightarrow\left(\frac{H^{d-1}\left(\bar{X}_{\bar{\eta}}, \mathcal{K}_{d}\right)}{\bar{\jmath}^{*} H^{d-1}\left(\bar{X}, \mathcal{K}_{d}\right)}\right)^{\Gamma}\right]
$$

Then, applying the snake lemma to the preceding diagrams, we obtain ${ }^{1}$

Proposition 3.1. There exists a natural exact sequence

$$
\begin{aligned}
& \bigoplus_{y \in C_{0}} C H^{d-1}\left(X_{y}\right)^{\prime} \rightarrow \operatorname{Ker}\left[C H^{d}(X)^{\prime} \rightarrow C H^{d}\left(X_{\eta}\right)^{\prime}\right] \\
& \rightarrow \operatorname{Ker}\left[E(\bar{X} / \bar{C}) \rightarrow \bigoplus_{y \in C_{0}} \frac{C H^{d-1}\left(\bar{X}_{\bar{y}}\right)^{\Gamma_{y}}}{\pi_{\bar{y}}^{*} C H^{d-1}\left(X_{y}\right)}\right] \rightarrow 0,
\end{aligned}
$$

where $E(\bar{X} / \bar{C})$ is the group (3).

As regards the right-hand group in the exact sequence of the proposition, the following holds. Let

$$
H^{d-1}\left(X_{\eta}, \mathcal{K}_{d}\right)^{\prime}=\operatorname{Im}\left[H^{d-1}\left(X_{\eta}, \mathcal{K}_{d}\right) \rightarrow H^{d-1}\left(\bar{X}_{\bar{\eta}}, \mathcal{K}_{d}\right)^{\Gamma}\right]
$$

\footnotetext{
${ }^{1}$ Proposition 3.1 was inspired by [1, Proposition 1.1].
} 
and

$$
\operatorname{Sal}_{d}(X / C)=\left\{f \in H^{d-1}\left(\bar{X}_{\bar{\eta}}, \mathcal{K}_{d}\right)^{\Gamma}: \forall y \in C_{0}, \bar{\delta}_{\bar{y}}(f) \in \pi_{\bar{y}}^{*} C H^{d-1}\left(X_{y}\right)\right\},
$$

where $\bar{\delta}$ and $\pi_{\bar{y}}^{*}$ are the maps of diagram (2).

Proposition 3.2. There exists a natural exact sequence

$$
\begin{aligned}
0 & \rightarrow \frac{\operatorname{Sal}_{d}(X / C)}{\left(\bar{\jmath}^{*} H^{d-1}\left(\bar{X}, \mathcal{K}_{d}\right)\right)^{\Gamma} \cdot H^{d-1}\left(X_{\eta}, \mathcal{K}_{d}\right)^{\prime}} \\
& \rightarrow \operatorname{Ker}\left[E(\bar{X} / \bar{C}) \rightarrow \bigoplus_{y \in C_{0}} \frac{C H^{d-1}\left(\bar{X}_{\bar{y}}\right)^{\Gamma_{y}}}{\pi_{\bar{y}}^{*} C H^{d-1}\left(X_{y}\right)}\right] \\
& \rightarrow H^{1}\left(\Gamma, \bar{\jmath}^{*} H^{d-1}\left(\bar{X}, \mathcal{K}_{d}\right)\right) .
\end{aligned}
$$

Proof. This follows by applying the snake lemma to a diagram of the form

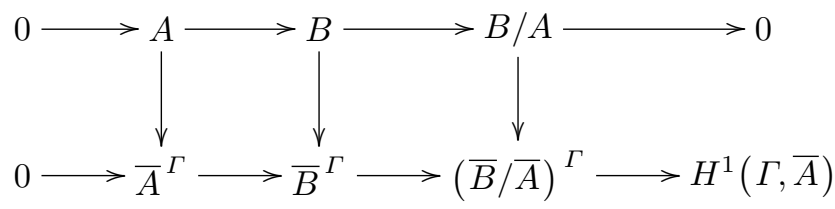

with $\bar{A}=\bar{\jmath}^{*} H^{d-1}\left(\bar{X}, \mathcal{K}_{d}\right), \bar{B}=H^{d-1}\left(\bar{X}_{\bar{\eta}}, \mathcal{K}_{d}\right)$, etc.

\section{Proof of the main theorem.}

Let $C$ and $A$ be as in Section 2, let $p$ be a prime number different from the characteristic of $k$ and let $X$ be a Severi-Brauer scheme over $C$ of relative dimension $p-1$.

Lemma 4.1. There exists a $\Gamma$-isomorphism

$$
\bar{\jmath}^{*} H^{d-1}\left(\bar{X}, \mathcal{K}_{d}\right) \simeq \bar{k}^{*} .
$$

Proof. Clearly, $\bar{\jmath}^{*} H^{d-1}\left(\bar{X}, \mathcal{K}_{d}\right)$ is the kernel of the map

$$
\bar{\delta}: H^{d-1}\left(\bar{X}_{\bar{\eta}}, \mathcal{K}_{d}\right) \rightarrow \bigoplus_{\bar{y} \mid y} C H^{d-1}\left(\bar{X}_{\bar{y}}\right)
$$

appearing in the exact sequence (1) over $\bar{k}$. Now $\bar{X}_{\bar{\eta}} \simeq \mathbb{P}_{\bar{\eta}}^{p-1}$ and $\bar{X}_{\bar{y}} \simeq \mathbb{P}_{\bar{k}}^{p-1}$ for every $\bar{y}$, whence we have $\Gamma$-isomorphisms

$$
H^{d-1}\left(\bar{X}_{\bar{\eta}}, \mathcal{K}_{d}\right) \simeq \bar{k}(C)^{*}
$$

and

$$
C H^{d-1}\left(\bar{X}_{\bar{y}}\right) \simeq \mathbb{Z}
$$

for each $\bar{y}$. Under these isomorphisms, the map $\bar{\delta}$ above corresponds to the canonical map

which yields the lemma.

$$
\begin{aligned}
& \bar{k}(C)^{*} \rightarrow \bigoplus_{\bar{y} \mid y} \mathbb{Z}, \\
& \left.f \mapsto \operatorname{ord}_{\bar{y}}(f)\right)_{\bar{y} \mid y},
\end{aligned}
$$


Theorem 4.2. For every $d$ such that $2 \leq d \leq p$, there exists a canonical isomorphism

$$
C H^{d}(X)^{\prime} \simeq C H_{0}(X / C) \text {. }
$$

Proof. By Lemma 4.1, Hilbert's Theorem 90 and Proposition 3.2, there exists a natural isomorphism

$$
\operatorname{Ker}\left[E(\bar{X} / \bar{C}) \rightarrow \bigoplus_{y \in C_{0}} \frac{C H^{d-1}\left(\bar{X}_{\bar{y}}\right)^{\Gamma_{y}}}{\pi_{\bar{y}}^{*} C H^{d-1}\left(X_{y}\right)}\right] \simeq \frac{\operatorname{Sal}_{d}(X / C)}{k^{*} H^{d-1}\left(X_{\eta}, \mathcal{K}_{d}\right)^{\prime}} .
$$

On the other hand, by $[7,(8.7 .2)], H^{d-1}\left(X_{\eta}, \mathcal{K}_{d}\right)^{\prime}=\operatorname{Nrd} A^{*}$ for every $d$ such that $2 \leq d \leq p$ and, for each $y \in C_{0}$,

$$
\pi_{\bar{y}}^{*} C H^{d-1}\left(X_{y}\right) \simeq \pi_{\bar{y}}^{*} C H^{p-1}\left(X_{y}\right) \simeq\left(q_{y}\right)_{*} C H_{0}\left(X_{y}\right) \quad(=\mathbb{Z} \text { or } p \mathbb{Z}) .
$$

The latter implies that $\operatorname{Sal}_{d}(X / C)=k(C)_{\mathrm{dn}}^{*}$, whence

$$
\begin{aligned}
\operatorname{Sal}_{d}(X / C) / k^{*} H^{d-1}\left(X_{\eta}, \mathcal{K}_{d}\right)^{\prime} & \simeq k(C)_{\mathrm{dn}}^{*} / k^{*} \operatorname{Nrd} A^{*} \\
& \simeq C H_{0}(X / C) .
\end{aligned}
$$

Finally, [loc.cit.] shows that the groups $C H^{d}\left(X_{\eta}\right)$ and $C H^{d-1}\left(X_{y}\right)\left(y \in C_{0}\right)$ are torsion free, whence $C H^{d}\left(X_{\eta}\right)^{\prime}$ and $C H^{d-1}\left(X_{y}\right)^{\prime}$ vanish. The theorem now follows from Proposition 3.1.

Corollary 4.3. Let $d$ be such that $2 \leq d \leq p$. Then $C H^{d}(X)^{\prime}$ is finite if

(1) $k$ is a number field, or

(2) $k$ is a field of finite type over $\mathbb{Q}, C=\mathbb{P}_{k}^{1}$ and $X$ has a 0 -cycle of degree one.

Proof. Indeed, in these cases the group $C H_{0}(X / C)$ is finite [4].

Corollary 4.4. In each of the cases listed in the previous corollary, the Chow ring $C H^{*}(X)$ is finitely generated as an abelian group.

Proof. The above corollary and Remark 2.1 show that $C H^{d}(X)$ is finitely generated for any $d$ such that $2 \leq d \leq p$. Since $C H^{0}(X)$ and $C H^{1}(X)=\operatorname{Pic}(X)$ are well-known to be finitely generated (see $[2, \S 1])$, the proof is complete.

Remark 4.5. The referee has suggested the following alternative approach to this paper.

Since there is only $p$-torsion in the Chow groups and $\operatorname{dim} X=p$, it is not difficult to relate the $E_{2}$ and $E_{\infty}$ terms in the Gersten-Quillen spectral sequence (see, e.g., [7, Proposition (8.6.2), p.320]). Hence if $K_{0}(X)$ is finitely generated, then the Chow groups of $X$ are also finitely generated. Now let $\Lambda$ be the Azumaya algebra over $C$ corresponding to the Severi-Brauer scheme $X \rightarrow C$ (see [6]). Then, by a well-known theorem of Quillen, $K_{0}(X) \simeq K_{0}(C) \oplus K_{0}(\Lambda)^{p-1}$. Hence if $K_{0}(C)$ and $K_{0}(\Lambda)$ are finitely generated, then the Chow groups of $X$ are also finitely generated. Now one can construct a commutative diagram with Swan's localization sequences for $\Lambda$ and $C$ and use it to relate the kernel of the restriction map from $K_{0}(\Lambda)$ to $K_{0}(C)$ (or $K_{0}(\bar{\Lambda})$ ) to the group $k(C)^{*} / k^{*} \operatorname{Nrd} A^{*}$. This gives more transparent proofs of the finiteness results and the introduction of the Azumaya algebra $\Lambda$ provides a natural explanation for the appearance of the group $k(C)^{*} / k^{*} \operatorname{Nrd} A^{*}$. 


\section{References}

[1] J. L. Colliot-Thélène and A. Skorobogatov, Groupe de Chow des zéro-cycles sur les fibrés en quadriques, K-Theory 7 (1993), 477-500.

[2] J. L. Colliot-Thélène, Cycles algébriques de torsion et $K$-théorie algébrique, In: Lecture notes in Math. 1553 (1991), Springer, New York, 1-49.

[3] J. L. Colliot-Thélène and W. Raskind, Groupe de Chow de codimension deux des variétés définies sur un corps de nombres: un théorème de finitude pour la torsion, Invent. Math. 105 (1991), $221-245$.

[4] E. Frossard, Groupe de Chow de dimension zéro des fibrations en variétés de Severi-Brauer, Comp. Math. 110 (1998), 187-213.

[5] W. Fulton, Intersection Theory, Second Ed. Springer-Verlag, 1998.

[6] A. Grothendieck, Le Groupe de Brauer I, In: Dix Exposés sur la Cohomologie des Schémas. North-Holland, Amsterdam, (1968), 46-66.

[7] A. S. Merkurjev and A. A. Suslin, $K$-cohomology of Severi-Brauer varieties and the norm residue homomorphism, Math. USSR Izv. 21 (1982), No. 2, 307-340.

[8] P. Salberger, Galois descent and class groups of orders, Lect. Notes in Math. 1142 (1985), 239-255.

[9] C. Sherman, Some theorems on the K-Theory of coherent sheaves, Comm. in Alg. 7 (1979), no. $14,1489-1508$

Departamento de Matemáticas, Universidad Andrés Bello, Chile

E-mail address: cristiangonzalez@unab.cl 